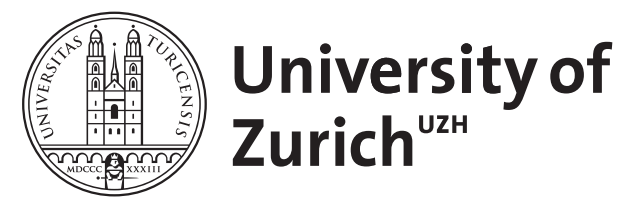

\title{
Retro-cue benefits in working memory without sustained focal attention
}

\author{
Rerko, Laura ; Souza, Alessandra S ; Oberauer, Klaus
}

\begin{abstract}
In working memory (WM) tasks, performance can be boosted by directing attention to one memory object: When a retro-cue in the retention interval indicates which object will be tested, responding is faster and more accurate (the retro-cue benefit). We tested whether the retro-cue benefit in WM depends on sustained attention to the cued object by inserting an attention-demanding interruption task between the retro-cue and the memory test. In the first experiment, the interruption task required participants to shift their visual attention away from the cued representation and to a visual classification task on colors. In the second and third experiments, the interruption task required participants to shift their focal attention within WM: Attention was directed away from the cued representation by probing another representation from the memory array prior to probing the cued object. The retro-cue benefit was not attenuated by shifts of perceptual attention or by shifts of attention within WM. We concluded that sustained attention is not needed to maintain the cued representation in a state of heightened accessibility.
\end{abstract}

DOI: https://doi.org/10.3758/s13421-013-0392-8

Posted at the Zurich Open Repository and Archive, University of Zurich ZORA URL: https://doi.org/10.5167/uzh-97058

Journal Article

Published Version

Originally published at:

Rerko, Laura; Souza, Alessandra S; Oberauer, Klaus (2014). Retro-cue benefits in working memory without sustained focal attention. Memory Cognition, 42(5):712-728.

DOI: https://doi.org/10.3758/s13421-013-0392-8 


\title{
Retro-cue benefits in working memory without sustained focal attention
}

\author{
Laura Rerko • Alessandra S. Souza • Klaus Oberauer
}

Published online: 18 January 2014

(C) Psychonomic Society, Inc. 2014

\begin{abstract}
In working memory (WM) tasks, performance can be boosted by directing attention to one memory object: When a retro-cue in the retention interval indicates which object will be tested, responding is faster and more accurate (the retro-cue benefit). We tested whether the retro-cue benefit in WM depends on sustained attention to the cued object by inserting an attention-demanding interruption task between the retro-cue and the memory test. In the first experiment, the interruption task required participants to shift their visual attention away from the cued representation and to a visual classification task on colors. In the second and third experiments, the interruption task required participants to shift their focal attention within WM: Attention was directed away from the cued representation by probing another representation from the memory array prior to probing the cued object. The retro-cue benefit was not attenuated by shifts of perceptual attention or by shifts of attention within WM. We concluded that sustained attention is not needed to maintain the cued representation in a state of heightened accessibility.
\end{abstract}

Keywords Working memory $\cdot$ Retro-cue $\cdot$ Attention · Bindings

When asked to briefly memorize an array of visual objects for a subsequent recognition test, performance is improved by providing a cue that indicates the location of the object to be tested. This cueing benefit can be observed when the cue is provided prior to the presentation of the memory array, the so-called precue (Griffin \& Nobre, 2003; Nobre et al., 2004; Posner, 1980; Schmidt, Vogel, Woodman, \& Luck, 2002; Woodman, Vecera, \& Luck, 2003). The cueing benefit is also observed

L. Rerko $(\bowtie) \cdot$ A. S. Souza $\cdot$ K. Oberauer

Department of Psychology, Cognitive Psychology Unit, University

of Zürich, Binzmühlestrasse 14/22, 8050 Zürich, Switzerland

e-mail: 1.rerko@psychologie.uzh.ch when the cue is presented after the offset of the memory array, the so-called retro-cue (Griffin \& Nobre, 2003; Hollingworth \& Maxcey-Richard, 2013; Kuo, Stokes, \& Nobre, 2012; Landman, Spekreijse, \& Lamme, 2003; Lepsien \& Nobre, 2007; Makovski \& Jiang, 2007; Makovski, Sussman, \& Jiang, 2008; Matsukura, Luck, \& Vecera, 2007; Maxcey-Richard \& Hollingworth, 2013; Nobre, Griffin, \& Rao, 2008; Sligte, Scholte, \& Lamme, 2008). Cueing improves accuracy by about $5 \%$ to $15 \%$ and speeds up reaction times by about $100-200 \mathrm{~ms}$ for cued trials, as compared to trials with a noninformative cue or without a cue (cf. Griffin \& Nobre, 2003).

Most explanations of the retro-cueing benefit rest on the assumption that the retro-cue guides focused attention to the memory object in the cued location, and that focal attention persistently stays on that object until the time of testing, thereby keeping it in a state of heightened accessibility (e.g., Makovski \& Jiang, 2007; Makovski et al., 2008; Matsukura \& Hollingworth, 2011; Matsukura et al., 2007; Pertzov, Bays, Joseph, \& Husain, 2013). A recent study by Hollingworth and Maxcey-Richard (2013) has called this assumption into question. They tested whether sustained visual attention is a prerequisite for the retro-cue benefit. They presented two kinds of cues during the retention interval of visual recognition task: a noninformative cue (i.e., one that provided no information regarding the object to be tested) or a valid retro-cue. Additionally, on a subset of trials an interruption task was presented after the cue and prior to the probe array. The interruption was a visual-search task that required participants to search for a target stimulus among distractors. The retro-cue benefit for memory performance was not attenuated by the interruption task. This finding shows that shifting perceptual attention away from the retro-cued object does not change its privileged state in working memory (WM), and hence that sustained spatial attention to the retro-cued object is no prerequisite for the retro-cue benefit (Hollingworth \& Maxcey-Richard, 2013). 
Hollingworth and Maxcey-Richard (2013) established that the retro-cue benefit is robust against distraction of spatial attention. In the present study, we investigated whether the retro-cue benefit requires sustained feature-based attention (Exp.1) or sustained attention within WM (Exps. 2 and 3). It is possible that visual search engages primarily attention to stimuli in spatial locations, whereas attention to objects in WM engages feature-based attention (i.e., attention to visual features of the cued objects, such as their color or their orientation). In Experiment 1, we tested whether shifting feature-based attention away from the cued representation to perform a perceptual classification task would disrupt the retro-cue benefit. Alternatively, it is possible that attention to perceptual information is separate from attention to information in WM. In Experiments 2 and 3, we tested whether shifting attention away from the cued representation to retrieve another object from the memory array would disrupt the retro-cue benefit.

To clarify, our research addressed the question of whether attention directed selectively on the cued object in WM needs to remain focused on that object throughout the retention interval. We were not concerned with the question of whether maintaining multiple objects in WM requires attention. Some theories of WM assume that maintaining a set of objects in WM requires a broad focus of attention that is assumed to hold up to about four independent chunks (Cowan, 2005), or requires executive attention (Kane \& Engle, 2002). These forms of attention are assumed to be responsible for holding the entire memory set (or whatever part of that set people can hold in WM), so it cannot at the same time be responsible for selectively improving accessibility of one object within that set in response to a retro-cue. When we speak of (focused) attention in the context of this article, we refer to the attentional mechanism that selectively focuses the retro-cued object.

\section{Experiment 1}

To investigate whether the retro-cue benefit depends on sustained attention to perceptual features, we independently manipulated whether or not a retro-cue was presented, and whether or not a perceptual interruption task occurred during the retention interval of a visual recognition task. In the recognition task, six colored discs had to be remembered over a retention interval. At the end of this interval, a probe stimulus was shown in one of the locations previously occupied by a memory object, and participants had to decide whether the color of the probe matched the color of the memory object presented in the same location. This paradigm has been referred to as a single-probe change detection task (Wheeler \& Treisman, 2002) or as a local-recognition task (Oberauer, 2003). In retro-cue trials, an informative retro-cue (i.e., a central arrow pointing from the center of the screen to the location that would be probed) was presented during the retention interval, whereas in no-cue trials, none of the objects in the memory array was cued. Orthogonal to the retro-cue manipulation, we varied whether or not a brief interruption task was performed during the retention interval (presented after the retro-cue in retro-cue trials). The interruption task consisted of a binary color classification task. Therefore, this task required feature-based attention to a feature on the same feature dimension that discriminated the objects in the memory array.

We expected to observe better performance in retro-cue trials than in no-cue trials in conditions without the interruption task, thus replicating the standard retro-cue benefit (e.g., Griffin \& Nobre, 2003; Makovski \& Jiang, 2007, 2008). If sustained feature-based attention is required for the retro-cue benefit, we expected this benefit to vanish or to be significantly reduced for interrupted trials. However, if sustained featurebased attention is not a prerequisite for a retro-cue benefit, comparable benefits should be observed with and without a perceptual interruption.

\section{Method}

Participants A group of 19 students participated in Experiment 1 (mean age 23 years, range 18-32) and received financial compensation or course credit. One participant was excluded due to performance at chance level, and one participant was excluded due to technical problems. For all of the experiments reported in this article, participants read and signed an informed consent form prior to the experiment. After the experiment, they were debriefed regarding the purpose of the study.

Materials and procedure All of the experiments reported here were programmed in MATLAB using the Psychophysics Toolbox (Brainard, 1997; Pelli, 1997).

Participants performed a visual recognition task. In the beginning of each trial, a set of six colored discs (memory array) was presented against a gray background for $1 \mathrm{~s}$. The discs were arranged at equal distances on an imaginary circle around the center of the screen. The memory array was followed by a blank retention interval (for the lengths of intervals, see Fig. 1). In the retro-cue conditions, $700 \mathrm{~ms}$ after the offset of the memory array, a white arrow extending from the center of the screen (retro-cue) was presented for $100 \mathrm{~ms}$. The retro-cue validly indicated which object from the memory array was to be probed later. At the end of each trial, a probe stimulus was shown in one of the positions previously occupied by a memory object in no-cue trials, or in the retro-cued location in retro-cue trials. The probe stimulus remained on screen until participants answered whether the probe color matched the color of the memory object that had been 

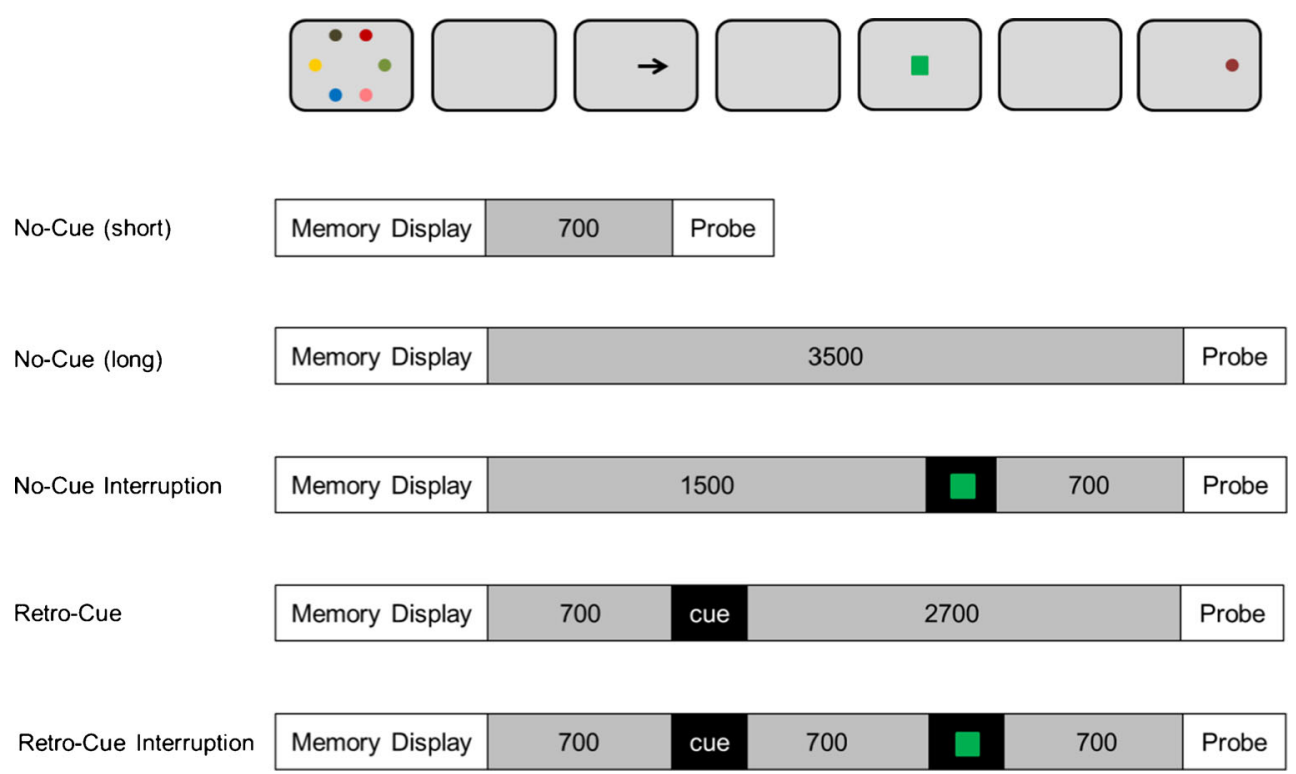

Fig. 1 Depiction of the flow of events (top row) and timing of events (following rows) for each condition of Experiment 1 . At the beginning of a trial, participants saw an array of six colored discs for 1,000 ms. After a retention interval, a probe stimulus was displayed in one of the locations previously occupied by a memory object, and participants had to judge whether the probe color matched the color of the object presented at the

presented in the same position. In the case of a match, participants had to press the left arrow key, and in the case of a mismatch, the right arrow key. Mismatch trials were equally divided into trials with a new color probe (i.e., a color that has not been part of the current memory array) and intrusion color probes (a color that has been part of the current memory array, but was not presented at the probed location). Feedback was displayed visually for $500 \mathrm{~ms}$ and was followed by a blank gray screen for $1.5 \mathrm{~s}$ before the next trial began. Participants were instructed to answer as quickly as possible without making mistakes.

In the interruption conditions, a speeded perceptual binary choice task was embedded during the retention interval of the visual recognition task. The interruption task was always presented $1,500 \mathrm{~ms}$ after the offset of the memory array (therefore, $700 \mathrm{~ms}$ after the offset of the retro-cue in retrocue trials). The interruption task comprised the classification of a centrally presented colored rectangle as warm (shades of red, yellow, pink, and brown) or cold (shades of blue, green, and violet). A total set of 14 colors (half of them warm and half of them cold) was used for the interruption task. Ten of these colors were the same colors as the ones used for the visual recognition task (overlapping colors), and four colors (half of them warm) were only presented during the interruption task, but could never appear in a memory array (nonoverlapping colors). Participants were not informed about this manipulation. The colors assigned to each set (i.e., overlapping and nonoverlapping) were randomly selected for each participant. The interruption stimulus was chosen from the same location. In retro-cue trials, a retro-cue was displayed for $100 \mathrm{~ms}$, $700 \mathrm{~ms}$ after the offset of the memory array. The retro-cue was a white arrow that extended from the center of the screen and pointed to the to-be probed location. In interruption trials, participants performed a color classification task during the retention interval (presented after the retrocue in retro-cue trials). The interruption task had a duration of $1.3 \mathrm{~s}$

overlapping set on half of the trials, and from the nonoverlapping set on the other half of trials.

Participants were instructed to respond as quickly and as accurately as possible to the interruption task by pressing the lower arrow key (warm colors) or the upper arrow key (cold colors). The interruption stimulus was presented at the center of the screen and remained there until participants entered their answer or until $1.3 \mathrm{~s}$ had elapsed. If an answer was given within the response window, a blank screen was shown until $1.3 \mathrm{~s}$ had passed from the onset of the interruption task. This enabled us to keep the overall retention intervals equal across conditions with and without interruptions (rows 3-6 in Fig. 1). If participants failed to respond within the response window, the trial was ended and discarded from the analysis. Participants were informed that every time they failed to respond within the response window, such that the trial had to be aborted, an additional delay would be inserted before the next trial started (the additional delay was $2.5 \mathrm{~s}$ ). This was done to discourage participants from taking the premature abortion of trials as a shortcut to end the experiment more quickly than usual.

We implemented two versions of the no-cue, nointerruption condition, one with a short retention interval, such that the time of probing matched the time of the retro-cue in the retro-cue condition, and another one with a long retention interval, such that the time of probing matched the time of probe onset in the remaining three conditions. The no-cue long condition served as our primary baseline to measure the retro-cue benefit and to assess whether this benefit vanished 
due to the interruption. This condition provided the same overall retention interval as the other conditions. It was possible, however, that in the retro-cue condition retrieval of the object to be probed would start not with the onset of the probe, but with the onset of the cue (Makovski et al., 2008), in which case the effective retention interval of the cued object would be shorter than in the no-cue trials with the same overall retention interval. To assess whether retro-cue benefits would still be obtained when the effective retention interval was the same across retro-cue and nocue trials, we also compared the retro-cue trials to the no-cue short condition.

In this experiment, participants completed two sessions. These trials were equally divided among the five conditions shown in Fig. 1 (80 trials per condition). Trials of each condition were randomly intermixed. Prior to the test trials, participants completed 40 practice trials on the interruption task alone, followed by 40 practice trials on the complete task in the first session. In the second session, these practice trials were reduced to four.

\section{Results}

Both reaction time (RT) and accuracy (percentage correct) served as dependent variables. The average accuracy in the WM task was $75.1 \%(S D=7.4)$, and the average accuracy in the interruption task was $80.9 \%(S D=12.1)$. Trials with errors in the interruption task were removed from all subsequent analyses. Furthermore, trials with errors in the memory task were excluded from the analysis of recognition RTs, as were trials with RTs faster than $200 \mathrm{~ms}$ and slower than $7 \mathrm{~s}$. This led to the exclusion of $30.5 \%$ of the RT data. The remaining RTs were logtransformed to reduce the skew of the distribution. In all graphical depictions of RT data throughout the article, RTs were trimmed (as described above) but not logtransformed, to enhance readability.

To analyze whether the retro-cue benefit remained even after feature-based attention was shifted away from the retrocue object in order to process a perceptual stimulus, we ran a repeated measures $2 \times 3$ analysis of variance (ANOVA) with the factors Cue (no-cue long vs. retro-cue) and Interruption (no interruption, interruption no-overlap, or interruption overlap). Figure 2 presents the means for these conditions, and Table 1 the ANOVA statistics. For accuracy, we observed a main effect of cue, showing higher accuracy in the retro-cue condition than in the no-cue condition. The main effect of interruption was significant as well, showing that responses were more accurate with no interruption that with interruption. However, the interaction between the two factors was nonsignificant, showing that the retro-cue benefits were of similar size in no-interruption and interruption trials. The retro-cue benefits were $18.5 \%(S D=7.5)$ in the no-interruption condition, $14.4 \%(S D=9.3)$ in the interruption no-overlap condition, and $20.4 \%(S D=8.5)$ in the interruption overlap condition. Paired $t$ tests showed that the retro-cue benefit in none of the interruption conditions differed significantly from the benefit in the no-interruption condition [interruption nooverlap condition, $t(16)=1.428, p=.173$; interruption overlap condition, $t(16)=0.797, p=.437]$. We also found a trend toward a larger retro-cue benefit in the interruption overlap condition than in the interruption nooverlap condition, $t(16)=2.005, p=.062$.

The RT analysis showed that participants responded faster in retro-cue trials than in no-cue trials. The effect of interruption was marginally significant, and the interaction was significant. The statistics from these analyses can be found in Table 1, and the means per condition are depicted in Fig. 2. Paired $t$ tests revealed that, relative to the no-interruption condition, mean benefit $=0.225, S D=0.066$, the retro-cue benefit was not reduced, but rather amplified by the interruption task, for both the interruption no-overlap condition, mean benefit $=$ $0.246 \mathrm{~s}(S D=0.091), t(16)=2.145, p=.048$, and the interruption overlap condition, mean benefit $=0.271 \mathrm{~s}(S D=$ $0.102), t(16)=2.477, p=.025$. The retro-cue benefits in the two interruption conditions did not differ significantly from each other, $t(16)=0.916, p=.373$.

For the no-cue short condition, the average percentage correct was $76.6 \%(S D=7.7)$, and the mean RT was $0.915 \mathrm{~s}(S D=0.159)$. The comparison of the no-cue short condition to the retro-cue conditions (no interruption, interruption no-overlap, and interruption overlap) provided significant retro-cue benefits for both percentages correct and RTs. The results of these comparisons can be found in Table 2. This finding shows that the retro-cue benefit is observed even relative to a no-cue condition in which the retention interval ends at the point in time when the retro-cue is presented in the cue conditions.

The results of Experiment 1 show that performance in the retro-cue task was not impaired by the intervening perceptual interruption task. To ensure that participants' performance in the interruption task was not impaired by retro-cueing, we conducted paired ttests comparing the performance in the interruption task across the no-cue and retro-cue conditions, separately for the two interruption types (overlap and no overlap). The tests confirmed that performance was not impaired in the retro-cue conditions relative to the condition in which no cue was provided. Indeed, for some comparisons, the opposite effect was found-namely, better performance on the interruption task in retro-cue trials than in no-cue trials. The results of these tests (for both percentages correct and RTs), including the means for each condition, can be found in Table 3 . 

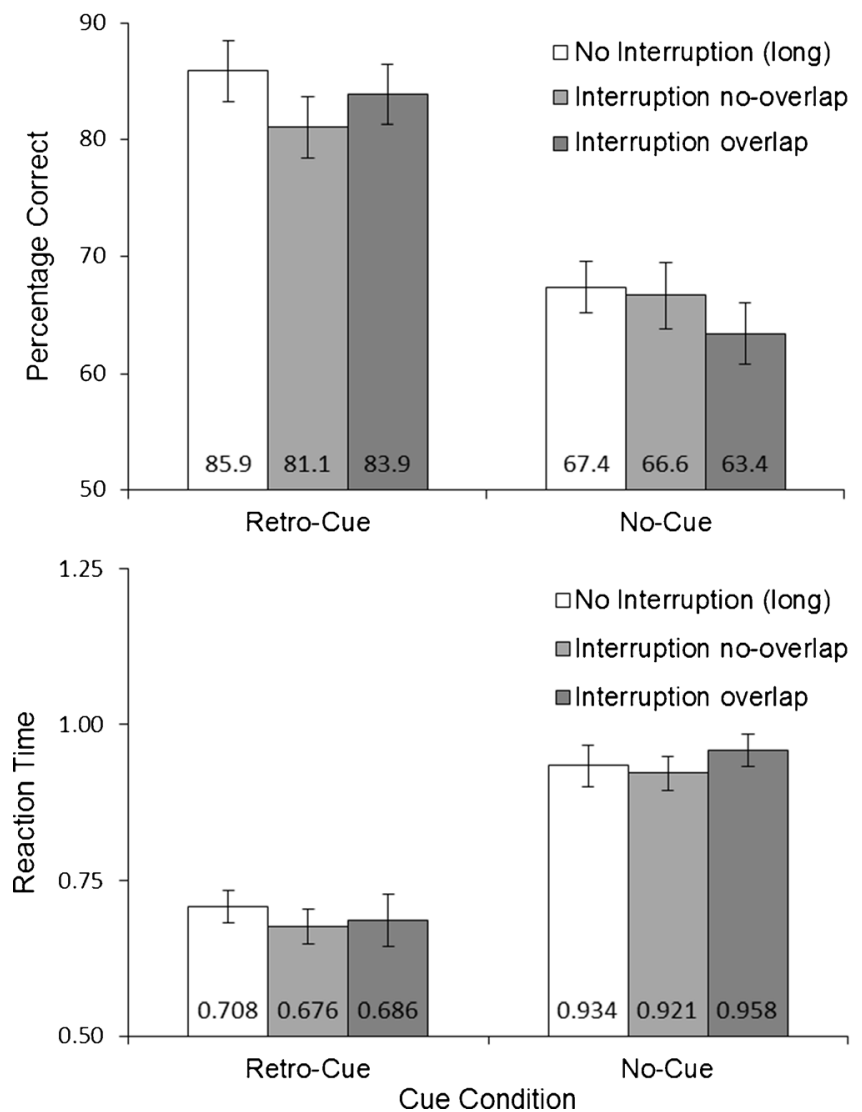

Fig. 2 Mean percentages correct (upper panels) and mean reaction times (lower panels) in Experiment 1, presented separately for each cue condition (retro-cue vs. no cue) and type of interruption (no interruption, interruption no-overlap, interruption overlap). The left panels depict

\section{Discussion}

Experiment 1 shows that the retro-cue benefit is not reduced when visual feature-based attention is shifted away from the retro-cued representation and directed to another object sharing the same feature as the memoranda. Attention could even be shifted to a feature from the set of features that could occur in the memory array without any attenuation of the retro-cue benefit.

Table 1 Results of the repeated measures Cue $\times$ Interruption ANOVA for Experiment 1 for percentages correct and log-transformed reaction times (RTs)

\begin{tabular}{llllll}
\hline Variable & $F$ Value & $d f 1$ & $d f 2$ & Partial $\eta^{2}$ & $p$ \\
\hline Percentage Correct & & & & & \\
$\quad$ Cue & 191.798 & 1 & 16 & .923 & $<.001$ \\
$\quad$ Interruption & 4.471 & 2 & 32 & .218 & .019 \\
$\quad$ Cue $\times$ Interruption & 2.450 & 2 & 32 & .133 & .102 \\
RT (log-transformed) & & & & & \\
$\quad$ Cue & 148.252 & 1 & 16 & .903 & $<.001$ \\
$\quad$ Interruption & 2.933 & 2 & 32 & .157 & .065 \\
$\quad$ Cue $\times$ Interruption & 3.933 & 2 & 32 & .197 & .030 \\
\hline
\end{tabular}
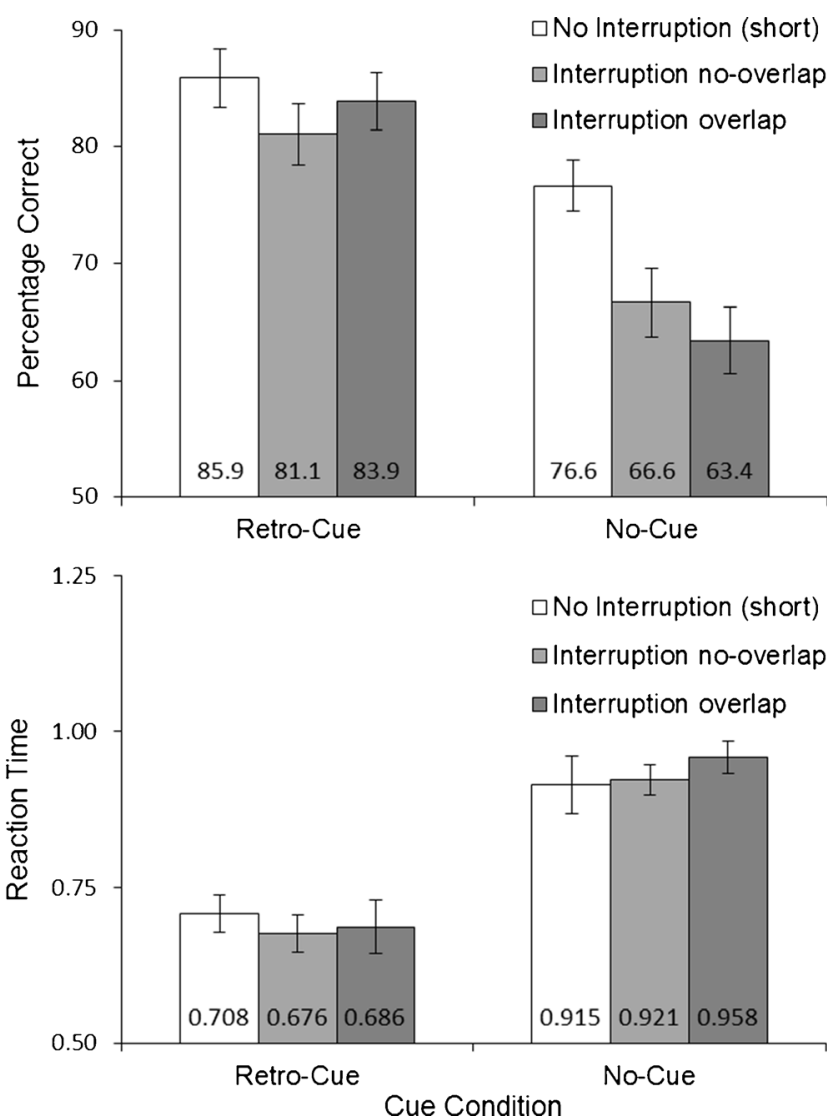

performance relative to the no-cue long, no-interruption condition, and the right panels relative to the no-cue short, no-interruption condition. Errors bars represent $95 \%$ within-subjects confidence intervals

In sum, Experiment 1 shows that the retro-cue benefit is neither abolished nor attenuated by perceptual interruptions that require feature-based attention to be shifted away from the cued representation. This finding extends the results reported by Hollingworth and Maxcey-Richard (2013): Whereas their experiments showed that sustained spatial attention is not required for the retro-cue benefit, the present Experiment 1 showed that sustained feature-based attention is not required, either.

Table 2 Results of the paired $t$ tests comparing the no-cue short condition to the retro-cue no-interruption and the retro-cue interruption conditions for Experiment 1 (for percentages correct and log-transformed RTs)

Variable

$t$ Value $d f p$

\section{Percentage Correct}

No-cue short vs. retro-cue, no interruption $\quad \begin{array}{llll}5.839 & 16 & <.001\end{array}$

No-cue short vs. retro-cue, interruption no-overlap $2.955 \quad 16 \quad .009$

No-cue short vs. retro-cue, interruption overlap $4.596 \quad 16<.001$

RT (log-transformed)

No-cue short vs. retro-cue, no interruption $\quad 7.872 \quad 16<.001$

No-cue short vs. retro-cue, interruption no-overlap $8.037 \quad 16<.001$

No-cue short vs. retro-cue, interruption overlap $\quad 7.276 \quad 16<.001$ 
Table 3 Results of the paired $t$ tests comparing the performance in the interruption task for the retro-cue interruption and the no-cue interruption conditions for Experiment 1 (for percentages correct and log-transformed RTs), separately for the no-overlap and overlap interruption conditions

\begin{tabular}{|c|c|c|c|c|c|}
\hline & Means & & & & \\
\hline & No Cue & Retro-cue & $t$ Value & $d f$ & $p$ \\
\hline \multicolumn{6}{|l|}{ Percentage Correct } \\
\hline Interruption no-overlap & $90.3(10.3)$ & $89.6(12.2)$ & 0.503 & 16 & .622 \\
\hline Interruption overlap & $79.2(14.5)$ & $82.4(12.3)$ & 2.532 & 16 & .022 \\
\hline \multicolumn{6}{|l|}{ RT } \\
\hline Interruption no-overlap & $0.828(0.078)$ & $0.813(0.090)$ & 1.639 & 16 & .121 \\
\hline Interruption overlap & $0.909(0.071)$ & $0.874(0.080)$ & 4.578 & 16 & $<.001$ \\
\hline
\end{tabular}

RT means are provided in seconds (nontransformed), and the corresponding ttests were calculated with the log-transformed RT data

Taken together, these results rule out the possibility that the retro-cue benefit requires sustained perceptual attention. It is still possible that attention to representations in WM is different from attention to perceptual information. Whereas several researchers have argued that attentional selection overlaps considerably in its behavioral as well as neurophysiological patterns in both WM and perception (Awh, Vogel, \& Oh, 2006; Griffin \& Nobre, 2003; Kuo, Rao, Lepsien, \& Nobre, 2009; Naghavi \& Nyberg, 2005; Olivers, 2008; Olivers, Meijer, \& Theeuwes, 2006), and some have even argued that the two forms of attentional selection are the same (Kiyonaga \& Egner, 2013); other authors have found evidence for only limited overlap between perceptual attention and attention to WM (Downing \& Dodds, 2004; Houtkamp \& Roelfsema, 2006; Makovski \& Jiang, 2007; Vogel, Woodman, \& Luck, 2001). Therefore, at present we cannot safely assume that attention to the contents of WM and perceptual attention are identical.

For that reason, we carried out Experiments 2 and 3 to test whether shifts of attention within WM rather than shifts of visual-perceptual attention reduce the retro-cue benefit. In these experiments, we required participants to shift their focus of attention in WM away from the retro-cued object to another object in memory by probing a noncued object prior to probing the cued one.

\section{Experiment 2}

Experiment 2 was motivated by theories that postulate a focus of attention in WM that selects a single object from the set of objects currently held in WM (Garavan, 1998; McElree, 2006; Oberauer, 2002, 2009; Olivers, Peters, Houtkamp, \& Roelfsema, 2011; for a review, see Oberauer \& Hein, 2012). Within these theories, the retro-cue benefit could be explained by assuming that the cued object is held in the focus of attention of WM until test. The focus of attention in WM could be independent of perceptual attention, and in that case one might not expect it to be distracted by an interruption task engaging perceptual attention. The focus of attention in WM would, however, be diverted from the retro-cued representation if it had to be directed to another representation in WM.

Therefore, in Experiment 2, we examined whether requiring the retrieval of a noncued object from WM reduces the retro-cue benefit for another, previously retro-cued object. We asked our participants to answer to two recognition probes from each memory array. On half of the trials, one object from the memory array was retro-cued during the retention interval, and the retro-cued object could be the first or the second to be probed. Critically, on trials in which the retro-cued object was the second to be probed, participants had to shift their focus of attention away from the retro-cued object to retrieve a noncued object in WM, in order to compare it to the probe stimulus and to select a response, before being probed for the retro-cued object. According to the theories cited above, the focus of attention selects only one object in WM at a time, so that the focus has to let go of the cued object when it is engaged in retrieving another object. This assumption is justified by the function of the focus of attention as a selection device: To select one object for retrieval, attention in WM needs to be focused exclusively on that object, to minimize confusions with other objects in WM (Oberauer \& Hein, 2012). If attention is focused on the probed but noncued object, it cannot at the same time be directed to the cued object-splitting attention among those two objects would create confusion between them at retrieval. If the retro-cue benefit survives the distraction of focal attention, we must conclude that the retro-cue benefit does not arise from holding the cued object in the focus of attention of WM. In contrast, if the benefit is abolished by this attentional shift, we could conclude that sustained focal attention in WM needs to be exclusively allocated to the cued representation in order to obtain a retro-cue benefit. 


\section{Method}

Participants A group of 24 students (mean age of 24 years, range 18-32) participated in this experiment. They received financial compensation or course credit for their participation.

Procedure The retro-cue paradigm employed in the present experiment was similar to the one used in Experiment 1, with the exception that two recognition probes were presented sequentially, testing two different objects instead of one (see Fig. 3). On half of the trials, a retro-cue was displayed $1 \mathrm{~s}$ after the offset of the memory array, and was followed $1 \mathrm{~s}$ later by the first probe stimulus. The retro-cue was visible for $100 \mathrm{~ms}$ and validly indicated one of the objects to be probed later. The other half of the trials comprised no-cue trials. These trials were equally split into a no-cue short condition and a no-cue long condition. In the no-cue short condition, the first probe was presented after a blank retention interval of $1 \mathrm{~s}$, corresponding to the onset time of the retro-cue in the retro-cue condition. In the no-cue long condition, the first probe appeared after an interval of $2.1 \mathrm{~s}$, matching the onset time of the first probe in the retro-cue condition. This first probe stimulus remained on screen until participants answered whether the probe color matched the color of the memory object presented at the same location. After the participant had responded to the first probe, the second probe was shown $100 \mathrm{~ms}$ later. Again, participants had to decide whether the probe color matched the color of the object presented in the same location in the memory array. For both probes, participants pressed the left arrow or the right arrow key to indicate a match or a mismatch response, respectively. Half of the trials were match trials, and the mismatch trials were equally divided into new-color probe trials and intrusion-color probe trials. Visual performance feedback for both probes was provided after the response to the second probe ("Response 1: correct/wrong; Response 2: correct/ wrong," in German) and remained on screen for $500 \mathrm{~ms}$. A blank intertrial interval of $1.5 \mathrm{~s}$ was provided before the next trial started.

The order of probing was manipulated block-wise: One block consisted of the no-cue trials randomly mixed with the retro-cue trials in which the cued object was always probed first (probed first). The other block comprised no-cue trials mixed with the retro-cue trials in which the retro-cued object was always probed second (probed second). The order of the two blocks was counterbalanced across participants. A short instruction prior to each block informed as to whether the retro-cued object would be probed first or second. Participants were instructed to answer as correctly and as quickly as possible.

Each participant completed one session $(75 \mathrm{~min})$ with a total of 480 trials ( 240 trials per experimental block). Half of the trials in each block comprised retro-cue trials, and the other half comprised no-cue trials. Before each block, participants completed 16 practice trials that were excluded from the subsequent analyses.

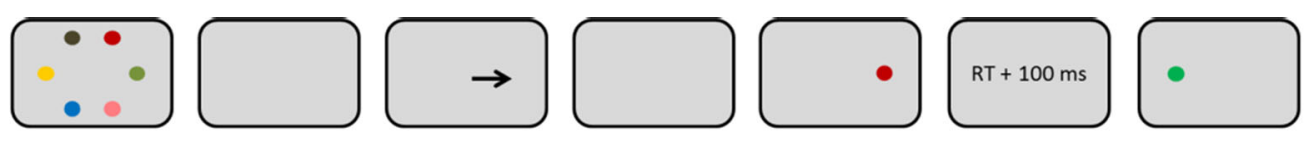

No-Cue (short)

\begin{tabular}{|l|l|l|l|}
\hline Memory Display & 1000 & 1st Probe & 2nd Probe \\
\hline
\end{tabular}

No-Cue (long)

\begin{tabular}{|l|l|l|l|}
\hline Memory Display & 2100 & 1st Probe & 2nd Probe \\
\hline
\end{tabular}

Retro-Cue probed first

\begin{tabular}{|l|l|l|l|l|l|}
\hline Memory Display & 1000 & cue & 1000 & 1st Probe (cued) & 2nd Probe (non-cued) \\
\hline
\end{tabular}

Retro-Cue probed second

\begin{tabular}{|l|l|l}
\hline Memory Display & $1000 \quad$ cue \\
\hline
\end{tabular}
1000 1st Probe (non-cued) 2nd Probe (cued)

Fig. 3 Flow of events (top row) and timing of events (following rows) for each condition of Experiment 2. At the beginning of a trial, participants saw an array of six colored discs for $1,000 \mathrm{~ms}$. At the end of a retention interval, two objects from the memory array were probed sequentially (the second probe was displayed $100 \mathrm{~ms}$ after the response to the first probe). In the retro-cue conditions, a retro-cue (duration = $100 \mathrm{~ms}$ ) was shown $1,000 \mathrm{~ms}$ after the offset of the memory array. The retro-cue validly indicated one of the objects that was going to be probed later. In the retro-cue conditions (rows 4 and 5), the retro-cued object and one of the noncued objects were probed sequentially. The order of probing was varied across conditions: Either the retro-cued object was probed first, followed by a probe testing one of the noncued objects (row 4; probed-first [no-interruption] condition), or one of the noncued objects was probed first, followed by the probe testing the retro-cued object (row 5 ; probed-second [interruption] condition) 


\section{Results and discussion}

The average percentages correct were $76.3 \%(S D=10.5)$ for answering the first probe and $70.7 \%(S D=10.0)$ for answering the second probe. RT data trimming and transformation was done as described for Experiment 1, separately for each probe. In total this led to an exclusion of $23.9 \%$ of RTs for the first probe and $31.4 \%$ of RTs for the second probe.

To examine whether the retro-cue benefit is reduced if the retro-cued object is tested after an intervening probe (i.e., a probe testing another, noncued object from the memory array), we ran $2 \times 2$ ANOVAs for the accuracy and logtransformed RT data with the factors Cue (no cue vs. retrocue) and Probe Order (probed first vs. probed second) as independent variables (average data are presented in Table 4 and Fig. 4). We ran two sets of analyses, separately contrasting the retro-cue trials to the no-cue short and no-cue long conditions. Both of these analyses revealed the same pattern of effects (see Table 5). The analyses for both dependent measures revealed a significant effect of cue, confirming that retrocue trials led to better accuracy and faster answers than did nocue trials (retro-cue benefit). The effect of probe order was significant, as well, showing that answers to the first probe were more accurate but slower than answers to the second probe. The interaction was not significant for the accuracy analysis, showing that the size of the retro-cue benefit was not affected by testing an intervening probe before testing the retro-cued object. However, for the RT data, the interaction was significant, showing a larger benefit when the retro-cued object was probed first than when the retro-cued object was probed second. A paired $t$ test showed that the retro-cue benefit in RTs was still significant after an intervening probe, for the comparisons with both the no-cue short and nocue long conditions, $t(23)=6.248, p<.001$, and $t(23)=6.238$, $p<.001$, respectively.

Could it be that, when the retro-cued object was probed second, people on some trials kept focusing the retro-cued object, sacrificing accuracy on the first-probed (noncued) object, and on other trials, shifted the focus to the firstprobed object, sacrificing the retro-cue benefit? In that case, there should be a trade-off between accuracy on the firstprobed and second-probed objects in the retro-cue condition. To test for such a trade-off, we computed the conditional probability of answering the second probe correctly, given that the first probe was answered correctly $(p 1)$, and given that the first probe was answered incorrectly $(p 0)$. We observed a trend toward higher values for $p 1$ (mean $=.793$, $S D=.118)$ than for $p 0$ (mean $=.752, S D=.134)$. This trend was not significant (after conversion of the probabilities into $z$ values), $t(23)=1.559, p=.133$, but it goes in the opposite direction from what would be expected from a trade-off between focusing on the cued object and focusing on the firstprobed object.

We also ran a $2 \times 2$ ANOVA to examine the effect of retrocueing an object on the retrieval of one of the noncued object in retro-cue trials, with the factors Cue (no cue vs. retro-cue, noncued probe) and Probe Order (probed first vs. probed second). For these analyses, we used the no-cue long condition as the baseline, because we investigate performance for

Table 4 Means and standard deviations (in parentheses) of the percentage-correct and RT (not log-transformed) data of Experiment 2, presented separately per block, cue condition, and probe order

\begin{tabular}{|c|c|c|c|c|c|}
\hline \multirow[b]{2}{*}{ Block } & \multirow[b]{2}{*}{ Condition: Probed Location } & \multicolumn{2}{|c|}{ Percentage Correct } & \multicolumn{2}{|c|}{ Reaction Time (s) } \\
\hline & & 1 & 2 & 1 & 2 \\
\hline \multirow[t]{2}{*}{1 st } & RC: Cued & $83.3(12.4)$ & - & $0.708(0.155)$ & - \\
\hline & NC short & $77.4(10.3)$ & $68.5(10.4)$ & $1.034(0.183)$ & $0.798(0.176)$ \\
\hline \multirow[t]{2}{*}{ 2nd } & RC: Cued & - & $78.0(12.2)$ & - & $0.652(0.156)$ \\
\hline & NC short & $77.0(12.4)$ & $68.5(9.1)$ & $1.075(0.259)$ & $0.815(0.197)$ \\
\hline \multirow[t]{2}{*}{$1 \mathrm{st}$} & RC: Cued & $83.3(12.4)$ & - & $0.708(0.155)$ & - \\
\hline & NC long & $74.5(10.4)$ & $69.4(11.3)$ & $1.037(0.247)$ & $0.834(0.207)$ \\
\hline \multirow[t]{2}{*}{ 2nd } & RC: Cued & - & $78.0(12.2)$ & - & $0.652(0.156)$ \\
\hline & NC long & $74.6(13.4)$ & $68.1(11.6)$ & $1.039(0.238)$ & $0.820(0.187)$ \\
\hline \multirow[t]{2}{*}{$1 \mathrm{st}$} & RC: Noncued & - & $67.6(11.6)$ & - & $0.883(0.255)$ \\
\hline & NC long & $74.5(10.4)$ & $69.5(11.3)$ & $1.037(0.247)$ & $0.834(0.207)$ \\
\hline \multirow[t]{2}{*}{2 nd } & RC: Noncued & $70.1(11.8)$ & - & $1.138(0.323)$ & - \\
\hline & NC long & $74.6(13.4)$ & $68.1(11.6)$ & $1.039(0.238)$ & $0.820(0.187)$ \\
\hline
\end{tabular}

$\mathrm{RC}:$ Cued = test of the retro-cued object in retro-cue trials; RC: Noncued = test of a noncued object in retro-cue trials; $\mathrm{NC}$ short = no-cue trial short; $\mathrm{NC}$ long = no-cue trial long. Standard deviations are provided in parentheses. Empty cells depict conditions that were not part of the experimental design (see Fig. 3). Conditions printed in bold were entered into the ANOVAs (separately for RTs and percentages correct) examining whether the retro-cue benefit was attenuated by an intervening probe. Conditions in italics were entered into the ANOVAs (separately for RTs and percentages correct) examining the effect of probing a retro-cued object prior to testing a noncued object. 

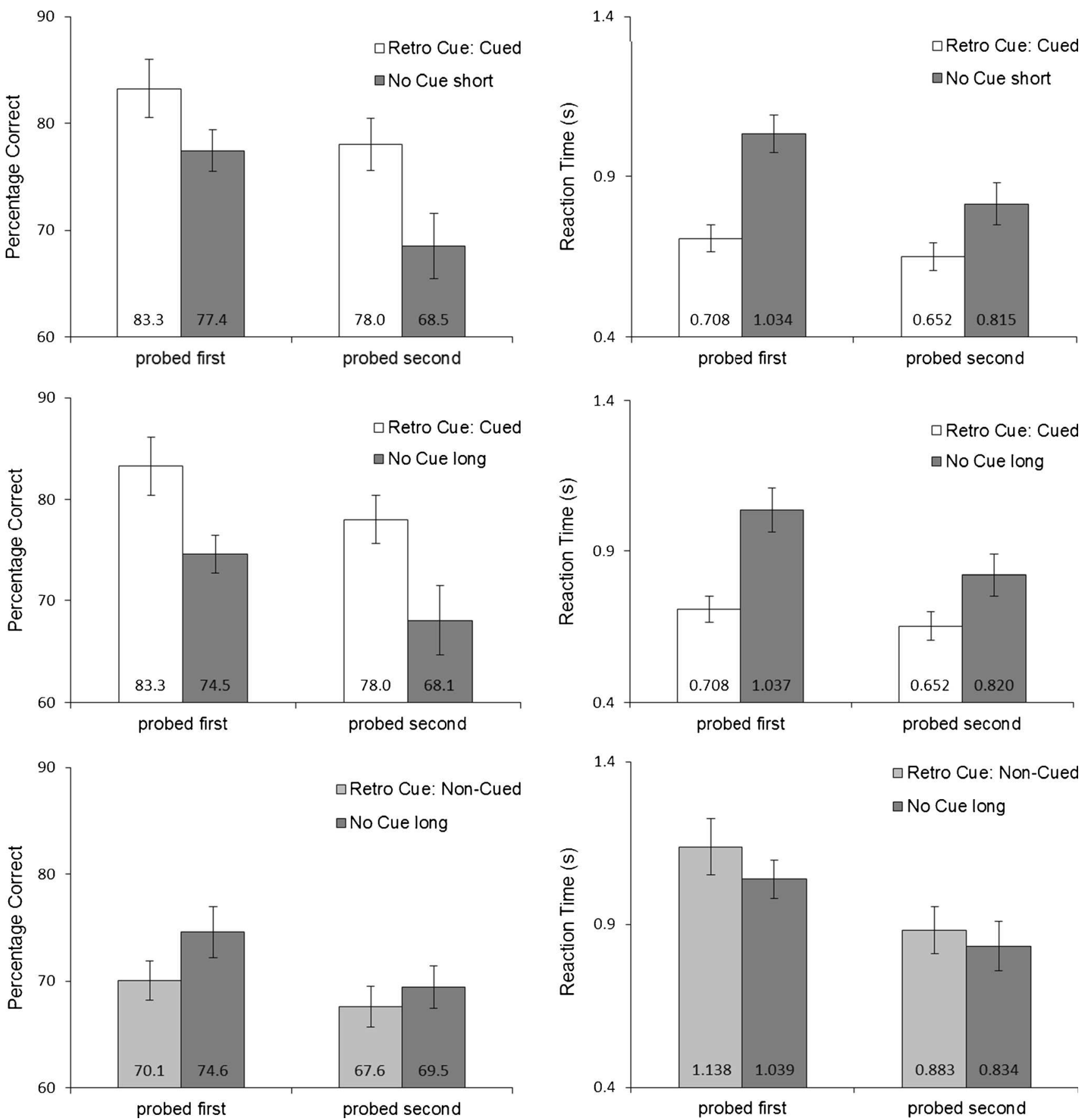

Fig. 4 Percentages correct (left panels) and mean nontransformed reaction times (right panels) to respond to probes in no-cue trials and retro-cue trials, as a function of probing order (probed first and probed second) in Experiment 2. The top row depicts responses to probes testing the retrocued object versus responses to probes in no-cue trials with a short retention interval. The middle row depicts responses to probes testing

noncued objects, for which the retention interval must have been the entire time between memory array and probe onset. The average data are shown in Table 4 and Fig. 4; the results of the ANOVA are presented in Table 5. The results show a significant main effect of cue: Performance on noncued the retro-cued object versus probes in the no-cue condition with a long retention interval. The bottom row depicts responses to probes testing one of the noncued objects in a retro-cue trial versus responses to probes in the no-cue condition with a long retention interval. Error bars represent $95 \%$ within-subjects confidence intervals

probes in the no-cue trials was better than in the retro-cue trials, revealing costs of probing another location than the cued one in retro-cue trials for both accuracy and RT. The main effect of probe order was significant, as well, showing that responding was more accurate, albeit slower, for the first 
Table 5 Results of the repeated measures Cue $\times$ Probe Order ANOVA on the percentage-correct and log-transformed RT data for Experiment 2

\begin{tabular}{|c|c|c|c|c|c|}
\hline Variable & $F$ Value & $d f 1$ & $d f 2$ & Partial $\eta^{2}$ & $p$ \\
\hline \multicolumn{6}{|c|}{$\begin{array}{l}\text { Retro-cued probe vs. No-cue short } \\
\text { Percentage Correct }\end{array}$} \\
\hline Cue & 39.340 & 1 & 23 & .631 & $<.001$ \\
\hline Probe Order & 19.362 & 1 & 23 & .457 & $<.001$ \\
\hline Cue $\times$ Probe Order & 2.901 & 1 & 23 & .112 & .102 \\
\hline \multicolumn{6}{|l|}{ RT (log-transformed) } \\
\hline Cue & 146.207 & 1 & 23 & .864 & $<.001$ \\
\hline Probe Order & 13.032 & 1 & 23 & .362 & .001 \\
\hline Cue $\times$ Probe Order & 36.189 & 1 & 23 & .611 & $<.001$ \\
\hline \multicolumn{6}{|c|}{$\begin{array}{l}\text { Retro-cued probe vs. No-cue long } \\
\text { Percentage Correct }\end{array}$} \\
\hline Cue & 51.674 & 1 & 23 & .692 & $<.001$ \\
\hline Probe Order & 14.479 & 1 & 23 & .386 & .001 \\
\hline Cue $\times$ Probe Order & 0.235 & 1 & 23 & .010 & .632 \\
\hline \multicolumn{6}{|l|}{ RT (log-transformed) } \\
\hline Cue & 116.388 & 1 & 23 & .835 & $<.001$ \\
\hline Probe Order & 10.780 & 1 & 23 & .319 & .003 \\
\hline Cue $\times$ Probe Order & 17.341 & 1 & 23 & .430 & $<.001$ \\
\hline \multicolumn{6}{|c|}{ Noncued probe vs. No-cue long } \\
\hline \multicolumn{6}{|l|}{ Percentage Correct } \\
\hline Cue & 19.062 & 1 & 23 & .543 & $<.001$ \\
\hline Probe Order & 8.029 & 1 & 23 & .259 & .009 \\
\hline Cue $\times$ Probe Order & 1.819 & 1 & 23 & .073 & .191 \\
\hline \multicolumn{6}{|l|}{ RT (log-transformed) } \\
\hline Cue & 19.620 & 1 & 23 & .460 & $<.001$ \\
\hline Probe Order & 17.091 & 1 & 23 & .426 & $<.001$ \\
\hline Cue $\times$ Probe Order & 0.262 & 1 & 23 & .011 & .614 \\
\hline
\end{tabular}

probe than for the second probe. The interaction was nonsignificant for both dependent measures, indicating that the retrocue costs were not affected by an intervening probe of a cued object.

The results of Experiment 2 show that the retro-cue benefit is not abolished by diverting focused attention to another object in WM before testing the cued object. Before discussing this result in detail, we report a third experiment, which addressed one limitation of Experiment 2.

\section{Experiment 3}

Unlike in Experiment 1, in Experiment 2 the durations of the retention interval for trials with and without an interruption by probing were not equal: Probing a noncued object prior to probing the retro-cued one prolonged the retention interval for testing the retro-cued object (interrupted trial; Fig. 3, row 5), relative to the retention interval when the retro-cued representation was tested first (noninterrupted trial; Fig. 3, row 4). In
Experiment 3, we equated these retention intervals by matching the onset time of the first probe in the noninterrupted trials to the onset time of the second probe in interrupted trials (see Fig. 5). We equated the retention intervals in these trials by inserting the average RT to respond to the first probe in the no-cue long condition in Experiment 2 to the postcue time of the retro-cue condition in which the retro-cued object was probed first, and also to the overall retention interval of a corresponding no-cue baseline condition (Fig. 5, rows 3 and 4). This manipulation produced approximately equal retention intervals for testing the noninterrupted retro-cue benefit (comparison of rows 3 and 4 in Fig. 5) and for testing the retro-cue benefit after an interruption (comparison of rows 2 and 5 in Fig. 5).

Method

Participants A group of 24 participants (mean age of 24 years, range 18-30 years) took part in Experiment 3. Two of them were excluded from the analyses due to poor performance 


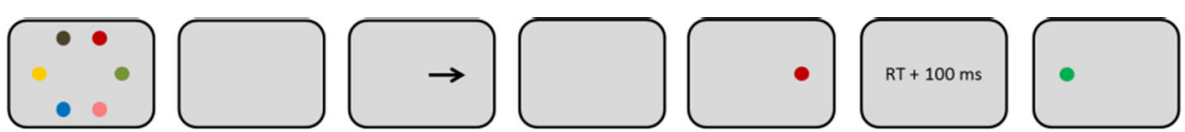

No Cue (short)

\begin{tabular}{|l|l|l|l|}
\hline Memory Display & 2100 & 1st Probe & 2nd Probe \\
\hline
\end{tabular}

No Cue (long)

\begin{tabular}{|l|l|l|l|l|}
\hline Memory Display & 2100 & mean RT + 100 & 1st Probe & 2nd Probe \\
\hline
\end{tabular}

Retro-Cue probed first

\begin{tabular}{|l|l|l|l|l|l|l|}
\hline Memory Display & 1000 & cue & 1000 & mean RT + 100 & 1st Probe (cued) & 2nd Probe (non-cued) \\
\hline
\end{tabular}

Retro-Cue probed second

\begin{tabular}{|l|l|}
\hline Memory Display & 1000 \\
\hline
\end{tabular}

cue

1000

1st Probe (non-cued)

2nd Probe (cued)
Fig. 5 Flow of events (top row) and timing of events (following rows) for each condition of Experiment 3. The task was to memorize an array of colored discs in order to respond to two sequentially presented probes (as in Exp. 2). In retro-cue trials, a retro-cue (duration= $100 \mathrm{~ms}$ ) was displayed 1,000 ms after the offset of the memory array. In the retro-cue conditions (rows 4 and 5), either the retro-cued object was probed first, followed by the test of a noncued object (row 4; probed first), or a noncued object was probed first, followed by the probe testing the retro-cued object (row 5; probed second). The conditions designed to

(50\% and $56 \%$ correct, on average, for answering the first and second probes correctly). ${ }^{1}$ Participants received financial compensation or participated in exchange for course credit.

Procedure The same basic procedure was employed as in Experiment 2. The critical variation from Experiment 2 to Experiment 3 concerned the timing of events in each condition. We implemented four conditions. Two conditions were identical to the ones employed in Experiment 2: (A) The nocue short condition (Fig. 5, row 2) corresponded to the no-cue long condition from Experiment 2 (Fig. 3, row 3), and (B) the retro-cue probed-second condition (Fig. 5, row 5) corresponded to the retro-cue probed-second condition in Experiment 2 (Fig. 3, row 5). In the remaining two conditions, the retention interval was prolonged, thereby creating $(C)$ a no-cue condition with a longer retention interval, no-cue long (see Fig. 5, row 3); and (D) a retro-cue probed-first condition with a longer postcue time (Fig. 5, row 4). These two conditions were prolonged in order to match the onset of the first probe in these conditions to the onset of the second probe in conditions $\mathrm{A}$ and $\mathrm{B}$ (Fig. 5, rows 2 and 5). In this way, we could compare the retro-cue benefits for a first-probed and a second-probed object at the same retention interval. To estimate by how much time conditions $\mathrm{C}$ and $\mathrm{D}$ had to be lengthened, we computed the mean RT to respond to the first

\footnotetext{
${ }^{1}$ The analyses reported were conducted on the data without these two participants. However, the pattern of effects was the same with and without these exclusions.
}

establish the retro-cue benefit for noninterrupted trials (i.e., trials in which the retro-cued object is probed first; rows 3 and 4) were prolonged so that the onset of the first probe in these trials matched the onset of the second probe in the interrupted trials (i.e., in which the retro-cued item was probed second; rows 2 and 5). The prolongation of the retention interval was achieved by inserting the mean RT $(1.1 \mathrm{~s})$ for responding to the first probe in the no-cue long condition in Experiment 2, plus the $100 \mathrm{~ms}$ of the interprobe interval

probe in the no-cue long condition from Experiment 2 $(\sim 1.1 \mathrm{~s})$. We then added the 100-ms interprobe interval of Experiment 2 to this average, resulting in a value of $1.2 \mathrm{~s}$.

The order of probing was manipulated block-wise: One block consisted of no-cue long trials and retro-cue trials in which the retro-cued object was probed first (Fig. 5, rows 3 and 4); the other block comprised nocue short trials and retro-cue trials in which the retrocued representation was probed second (Fig. 5, rows 2 and 5). Within each block, conditions were randomly intermixed. The order of blocks was counterbalanced across participants. A short instruction prior to each block informed as to whether the cued location would be probed first or second.

Each participant completed one session (approximately $80 \mathrm{~min}$ ), including 16 practice trials prior to each test block, which comprised 200 trials (100 per condition).

Results

The average percentage of correct responses to the first probe was $79.7 \%(S D=6.0)$, and to the second probe was $74.4 \%$ $(S D=6.4)$. The RT data were trimmed and log-transformed as in Experiment 2. This led to the exclusion of $20.3 \%$ of RTs for the first and $26.1 \%$ of RTs for the second probe.

In order to establish whether the retro-cue benefit survived the intervening probe, we ran a $2 \times 2$ ANOVA for both dependent measures with the factors Cue (no-cue vs. retro- 
cue) and Probe Order (probed first vs. probed second). Figure 6 depicts the means for the (nontransformed) RT and accuracy data for each experimental condition, and Table 6 shows the test statistics. The analyses revealed a main effect of cue, showing better performance and faster RTs for retro-cue trials than for no-cue trials. The effect of probe order was significant, as well, reflecting more accurate, but slower, responses for answering the first probe than for answering the second probe, replicating Experiment 2. No significant interaction was obtained for the accuracy data: The retro-cue benefit was not attenuated by an intervening probe. The interaction was significant for RT data, as in Experiment 2, showing a larger retrocue benefit for the first probe (noninterrupted trial) than for the second probe (interrupted trial). A paired ttest showed that the retro-cue benefit for the second probe was still significant, $t(21)=8.007, p<.001$.

As for Experiment 2, we compared whether performance on the second, retro-cued probe was better, depending on whether the first, noncued probe was answered correctly (trade-off between answering the first and the second probe). A paired $t$ test on the conditional probability of answering the second probe correctly, given that the first probe was answered correctly $(p 1)$ or incorrectly $(p 0)$, revealed higher values for $p 1$ (mean $=.848, S D=.079$ ) than for $p 0($ mean $=.811, S D=.104)$. Although this trend was not significant (after conversion of the probabilities into $z$ values), $t(21)=1.117, p=.126$, it indicates even better performance on the second, retro-cued probe when the first one was answered correctly than when it was answered incorrectly. This finding does not support the assumption of a trade-off.

We ran the same $2 \times 2$ ANOVA as for Experiment 2 to examine the effect of retro-cueing an object on the retrieval of a noncued object in retro-cue trials with the factors Cue (no cue vs. noncued item in the retro-cue condition) and Probe Order (probed first vs. probed second). The means in each condition are shown in Fig. 6; the results of the ANOVAs are
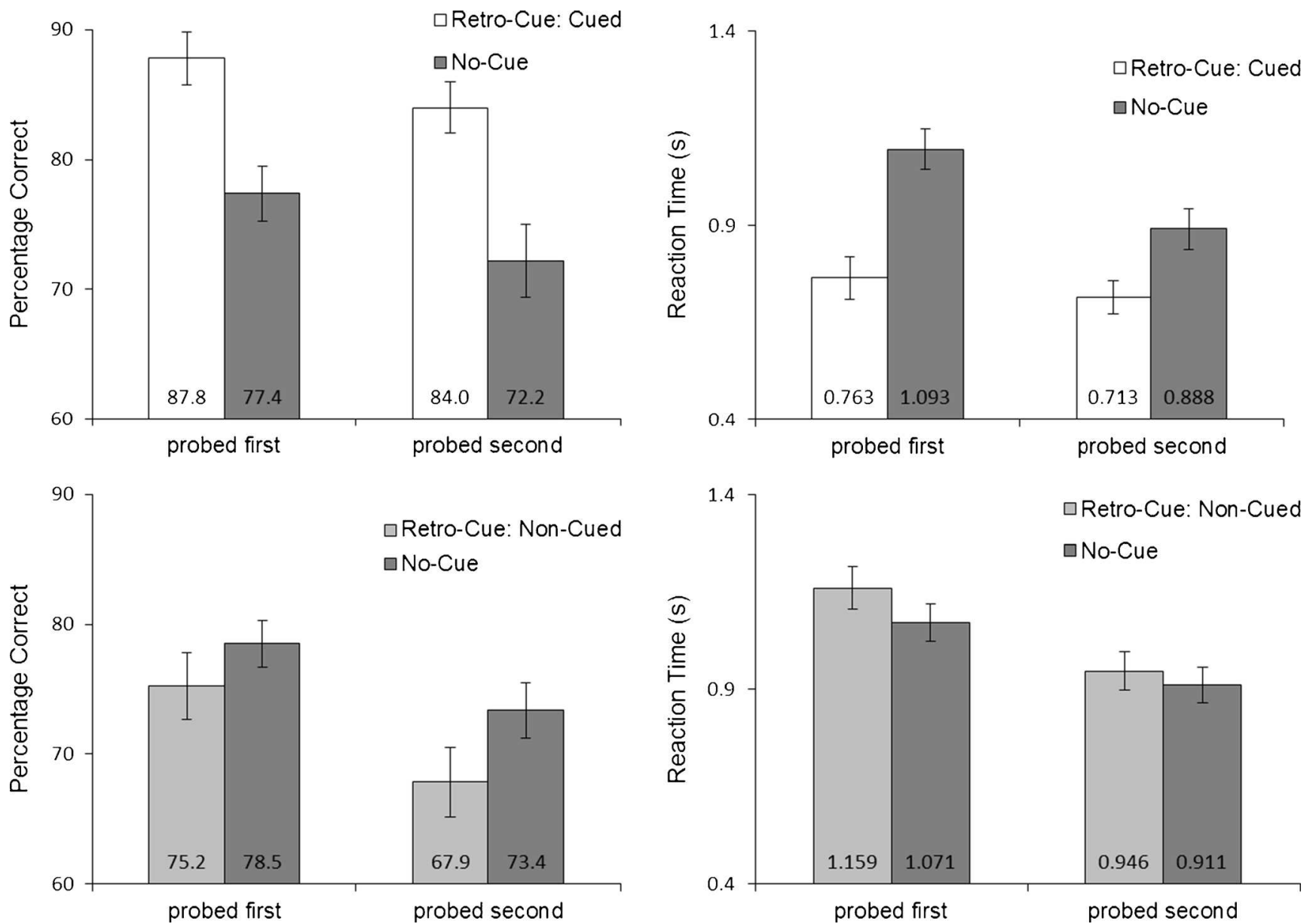

Fig. 6 Percentages correct (left panels) and mean nontransformed reaction times (right panels) to respond to probes in no-cue trials and retro-cue trials, as a function of probing order (probed first and probed second) in Experiment 3. The top row depicts responses to probes testing the retrocued object in retro-cue trials versus responses to probes in no-cue trials

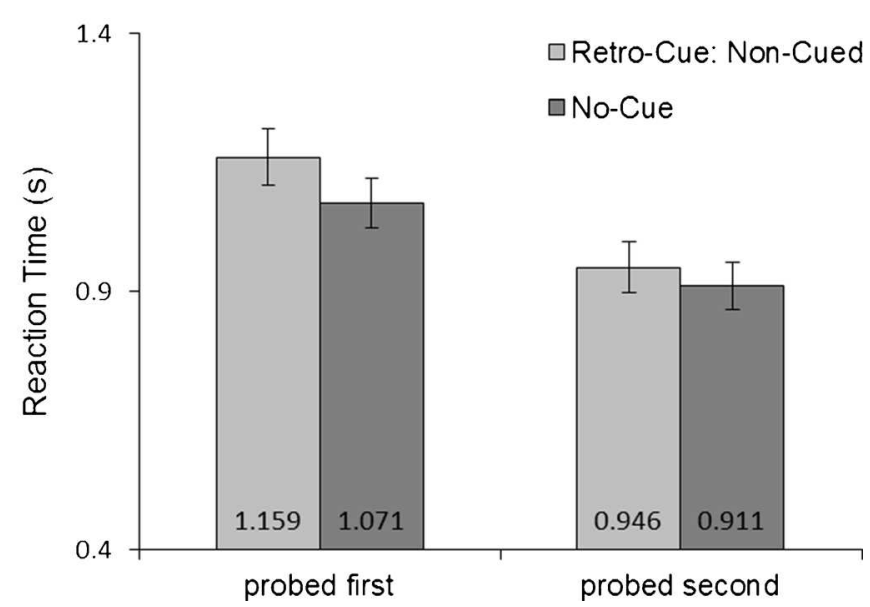

with the corresponding overall retention interval. The bottom row depicts responses to probes testing one of the noncued objects in retro-cue trials versus responses to probes in the corresponding no-cue trials. Error bars represent $95 \%$ within-subjects confidence intervals 
Table 6 Results of the repeated measures Cue $\times$ Probe Order ANOVA on the percentage-correct and log-transformed RT data for Experiment 3

\begin{tabular}{llllll}
\hline Variable & $F$ Value & $d f 1$ & $d f$ & Partial $\eta^{2}$ & $p$ \\
\hline $\begin{array}{l}\text { Cued probe vs. No-cue } \\
\text { Percentage Correct }\end{array}$ & & & & & \\
$\quad$ Cue & 66.548 & 1 & 21 & .760 & $<.001$ \\
$\quad$ Probe Order & 16.908 & 1 & 21 & .446 & $<.001$ \\
$\quad$ Cue $\times$ Probe Order & 4.99 & 1 & 21 & .023 & .488 \\
RT (log-transformed) & & & & & \\
$\quad$ Cue & 152.963 & 1 & 21 & .879 & $<.001$ \\
$\quad$ Probe Order & 13.062 & 1 & 21 & .383 & .002 \\
$\quad$ Cue $\times$ Probe Order & 21.704 & 1 & 21 & .508 & $<.001$ \\
Noncued probe vs. No-cue & & & & & \\
Percentage Correct & & & & & $<.001$ \\
$\quad$ Cue & 19.667 & 1 & 21 & .484 & \\
$\quad$ Probe Order & 21.111 & 1 & 21 & .501 & $<.001$ \\
$\quad$ Cue $\times$ Probe Order & 0.891 & 1 & 21 & .041 & .356 \\
RT (log-transformed) & & & & & \\
$\quad$ Cue & 18.018 & 1 & 21 & .462 & $<.001$ \\
Probe Order & 27.406 & 1 & 21 & .566 & $<.001$ \\
Cue $\times$ Probe Order & 2.662 & 1 & 21 & .112 & .118 \\
\hline
\end{tabular}

presented in Table 6. We found main effects of cue for both RTs and accuracy, revealing costs of cueing on retrieving a noncued item: Testing of a noncued object led to worse performance than did responding to a probe in a no-cue trial in which none of the items were cued. The main effect of probe order showed that responding was more accurate but slower for the first than for the second probe, in line with the results obtained for the same analysis in Experiment 2. The interaction was nonsignificant for both dependent measures, showing that the retro-cue costs were not affected by probing a noncued representation prior to probing the cued object. In sum, Experiment 3 replicated all of the findings from Experiment 2 while holding the retention interval constant for objects probed first and objects probed second.

\section{Discussion of Experiments 2 and 3}

The retro-cue benefit for accuracies was not diminished by testing an intervening noncued probe, and numerically we even observed a trend toward a larger benefit when an intervening probe preceded the test of the retro-cued object. This pattern of effects was obtained in both Experiment 2, in which the retention interval was shorter for probing the cued representation first than for probing the cued representation after an intervening probe, and Experiment 3, in which we matched the onsets of the probes testing the retro-cued object either first or second. Hence, both experiments support the conclusion that the retro-cue benefit does not require sustained focal attention to the cued object in WM.

In the RT analysis, the retro-cue benefit was attenuated but not abolished by the intervening probe. This attenuation can be explained as follows: The cue draws the focus of attention to the cued object. When the cued object is probed first, the focus of attention is already on the probed object, so that retrieval can commence immediately, without the need to shift the focus to the relevant item. In contrast, when the cued object is probed second, the focus of attention needs to shift from the cued object to the first-probed (noncued) object, and then needs to shift back from the first-probed object to the cued object. The shift back costs extra time that is added to the measured RT, and therefore diminishes the retro-cue benefit in RTs. Nevertheless, the cued object remains in a state of heightened accessibility while the noncued object is retrieved, so that the retro-cue benefit on accuracy is undiminished by the intervening retrieval of another object. Therefore, the reduction of the retrocue benefit in RTs, but not in accuracy, lends support to our conclusion that probing a noncued object prior to the cued object did indeed temporarily divert the focus of attention from the cued object.

The main effects of probe order show that responses were more accurate but slower for the first than for the second probe. This pattern matches findings from verbal WM (Cowan, Saults, Elliot, \& Moreno, 2002; Oberauer, 2003) and visual WM (cf. Woodman \& Vecera, 2011). The decline in accuracy over successive probes reflects output interference. The longer RTs for the first probe could reflect additional time needed to switch from memory encoding and maintenance to retrieval. Alternatively, the faster RTs in response to the second probe could reflect the fact that each probed object is subsequently being removed from WM, thereby reducing the number of competitors for subsequent retrievals (Farrell \& Lewandowsky, 2012).

We also examined the effects of cueing one representation in WM on the noncued representation. Probing a noncued object in a retro-cue trial was costly (reflected in worse accuracy and slower RTs), relative to a trial in which no object was cued. This finding is in line with the finding of invalid-cueing costs: In the classic retro-cue paradigm, if a location other than the retro-cued location is probed on a small proportion of trials, performance is impaired relative to a no-cue or noninformative-cue condition (e.g., Astle, Summerfield, Griffin, \& Nobre, 2012; Griffin \& Nobre, 2003; Pertzov et al., 2013). One plausible explanation for the retro-cue cost when probing a noncued representation is that when object $\mathrm{A}$ is retro-cued, and then object B is tested, retrieval of object B suffers from increased interference from the strong competitor A. This explanation builds on the assumption that object A has increased accessibility during retrieval of object $\mathrm{B}$, thereby 
impairing retrieval of object $B$, as was indicated by our findings. ${ }^{2}$ Another possibility is that the noncued items are partially removed from WM, and therefore when one of the noncued items is probed, this object is less accessible. Our results further show that the retro-cue costs were unaffected by the order of probing. This means that a cost is also observed when object $A$ is cued and probed first, and then a noncued object B is probed. According to the strong-competitor hypothesis, this would imply that object A retains its status of heightened accessibility (relative to the no-cue control condition) after being probed. This finding is furthermore in line with the removal assumption, as it would be expected that the removed information would be difficult to access as soon as it was removed, independent of whether or not another object was probed before probing one of the removed objects.

In sum, even an intervening probe diverting focal attention to a different memory object does not eliminate the benefits (and costs) of retro-cuing an object held in WM. This result rules out the hypothesis that the retro-cue benefit arises from maintaining this object persistently in the focus of attention in WM.

It might be argued that perhaps, when object $\mathrm{A}$ is retrocued and then a noncued object B is probed, the focus of attention partly but not fully concentrates on $\mathrm{B}$, and therefore retains A to some degree. This assumption faces two serious difficulties. First, it implies that when the cued object is probed second, the focus of attention must be shared between objects A and B, thereby leading to a reduction of the retrocue benefit when the cued object is probed second, relative to when it is probed first. This was not observed. Second, sharing the focus of attention between two objects raises the question of how a single object could be selectively retrieved from WM. Responding to the first probe required focusing attention on the probed object to select it from among the objects held in WM. It is difficult to conceive how focal attention could at the same time be maintained on another object, because doing so would undermine the selection function of focal attention: If object B is to be retrieved, and the retro-cued object A is being attended to at the same time, how could the WM system avoid erroneously retrieving object A? If both objects were in the focus of attention with equal status, then people would retrieve both of them with equal probabilities, and performance on probes of a noncued object in the retro-cue condition would be at chance, contrary to our results. If the focus of attention held both the cued object $\mathrm{A}$ and the probed object $\mathrm{B}$, but gave

\footnotetext{
${ }^{2}$ Previous research in our lab has shown that cueing one object does not impair access to other, noncued objects in a permanent, irrecoverable manner (Rerko \& Oberauer, 2013): When two different objects A and B are retro-cued one after another, and the second object (B) is tested, performance is as good as if that object had been the only one cued. Thus, when an object A is cued, another, noncued object B is difficult to retrieve when it is directly probed, but it can be recovered perfectly when it is retro-cued before being probed.
}

priority to $\mathrm{B}$, then that assignment of priority would be tantamount to focusing on $\mathrm{B}$ at the expense of $\mathrm{A}$, and the question would arise once again how the retro-cue benefit for A could be entirely unimpaired by the intervening retrieval of $\mathrm{B}$. The answer can only be that the retro-cue benefit does not depend on sustained focal attention to the cued object A. These considerations render the ad-hoc assumption of a focus of attention shared between two objects in WM unattractive. We therefore conclude-in line with the other experiment reported above - that no sustained focal attention is required in order to obtain a retro-cue benefit.

\section{General discussion}

We examined whether the retro-cue benefit depends on sustained attention allocated to the cued object. In three experiments we detracted attention from the cued representation in the interval between cue and probe. The retro-cue benefit survived distractions of feature-based visual attention to perceptual stimuli. This finding confirms and extends the results by Hollingworth and Maxcey-Richard (2013), who found no attenuation of the retro-cue benefit due to interruptions by a visual search task requiring visual-spatial attention.

We went one step farther, investigating whether the retrocue benefit might require sustained focal attention within WM, which might be distinct from perceptual attention. The retro-cue benefit was not abolished by probing a noncued representation between cue and probe. We conclude that a privileged representation in WM does not require sustained focal attention to maintain its privileged state. This conclusion converges with findings from a recent study by Rerko and Oberauer (2013): Multiple WM representations were cued sequentially, and the last one was always valid in predicting the location of the probe stimulus. When three representations were cued and the first and the third cued location were the same (cue sequence ABA), performance was better than when three different locations were cued (cue sequence CBA). This finding implies that the first-cued object retains privileged status while the focus of attention is directed to the secondcued object, so that when the last cue points again to the firstcued object (cue sequence ABA), its effect can build on the persisting effect of the first cue. Similarly, in a study by Maxcey-Richard and Hollingworth (2013), memory objects were presented sequentially, and cues indicating a higher or lower likelihood of probing were presented simultaneously or shortly after each corresponding memory object. Responding was more accurate to objects cued as being of higher probability than objects cued as having low probability, showing a cueing benefit. Importantly, this task required participants to encode new stimuli into WM while at the same time keeping a selected representation in a privileged status. Together with the results of the present study, we can conclude that cued 
representations can be maintained in a privileged state while information is either encoded into WM, as in Maxcey-Richard and Hollingworth's (2013) study, or retrieved from WM, as was shown in the present study.

In sum, we conclude that the maintenance of information in a highly accessible, privileged state in WM does not depend on sustained focal attention. Therefore, any successful explanation of the retro-cue benefit has to rely at least partly on other processes than focal attention.

If the retro-cue benefit does not emerge from the persistent deployment of attention to the retro-cued object, how can this benefit be explained? In the following, we outline three possible accounts for our finding within a theoretical framework that describes WM as consisting of three embedded components: The activated part of long-term memory, which keeps representations activated that might be needed for the current task; the region of direct access, which holds a limited amount of information in a highly accessible state and binds them into new structures; and the focus of attention, which is characterized as a selection device to single out individual objects (Oberauer, 2002, 2003, 2009; Oberauer \& Hein, 2012). According to this framework, the focus of attention is used to select one object in the region of direct access as input to the next cognitive action. One possible explanation for our findings within this framework is that the cue directs the focus of attention to the cued representation, and focal attention to the cued representation strengthens the binding between the content (e.g., color) and the context (e.g., location) of the cued representation (cf. Loaiza \& McCabe, 2012). Once this strengthened binding is established, it can be maintained in the region of direct access without sustained attention to the cued color or its location. As soon as the probe stimulus appears, focal attention is directed to the location of the probe, and the color bound to it is retrieved for comparison to the probe color. The retro-cue benefit arises because retrieval is facilitated for objects whose content-context bindings have been strengthened (cf. Rerko \& Oberauer, 2013).

A second alternative is that the retro-cue simply primes the context (e.g., a location) that is used to retrieve a representation from WM, not necessarily altering the strength of the bindings between content and context. Because the context serves as a retrieval cue, the corresponding color can be retrieved faster and more accurately than other, noncued objects. Both the cue-priming and the binding-strengthening mechanism are part of a computational model implementing the three-embedded-components framework (Oberauer, Souza, Druey, \& Gade, 2013).

A third possible explanation for our findings is removal of irrelevant information, a mechanism central to the SOB model ("serial order in a box"; Farrell \& Lewandowsky, 2002). According to this explanation, the retro-cue is used to distinguish between relevant and (temporarily) nonrelevant information, so that the nonrelevant information can be removed from the capacity-limited, central part of WM (i.e., the region of direct access) into the activated part of long-term memory (cf. Oberauer, 2005). Importantly, this information is not lost to WM, but can be brought back into the region of direct access if needed later (e.g., if cued later), as is shown by the reappearance of its behavioral effects (Oberauer, 2005) and of its neural signature (LaRocque, Lewis-Peacock, Drysdale, Oberauer, \& Postle, 2013; Lewis-Peacock, Drysdale, Oberauer, \& Postle, 2012). In the present experiments, the noncued objects could be removed from the direct-access region, thereby reducing interference for the cued object. If a noncued object needs to be retrieved before the cued object (Exps. 2 and 3), the noncued object needs to be brought back from activated LTM. Doing so is slower and less accurate than retrieval from the direct-access region, and therefore there is a cost of retro-cueing for noncued objects. At first glance, the removal explanation appears to be incompatible with the finding by Rerko and Oberauer (2013) that a cue sequence $\mathrm{ABA}$ results in better performance than does a CBA sequence. In fact, the removal explanation is perfectly compatible with this finding, if we consider that removal is a gradual, fairly slow process (Oberauer, 2002), so that it cannot be completed in the short time provided between two successive cues provided in the study by Rerko and Oberauer. Therefore, the third cue in the ABA sequence can still build on the residual representation of object $A$ that has not been entirely removed during the intervening cueing of $\mathrm{B}$, whereas the third cue in the CBA sequence points to an object that has been removed more completely.

Although all three explanations discussed above can account for the retro-cue benefit without requiring sustained focal attention to the cued representation, they do so by assuming different mechanisms for how this benefit is accomplished. Although both the strengthening and the cue-priming explanations rest on the assumption that the status of the cued representation is altered, by improving the accessibility of either its binding or its retrieval cue (in our experiments, the spatial location of the representation), the removal explanation attributes the retro-cue benefit not to a change of the cued representation, but to a status change of the noncued representations. According to the removal explanation, the cued representation remains essentially unchanged, and the removal of noncued information from the capacity-limited components of WM (e.g., region of direct access) reduces retrieval competition and interference, which in turn improves retrieval of the target representation (i.e., the cued representation).

One way to empirically distinguish these accounts would be to test a unique prediction of the removal hypothesis: Removing noncued information from the region of direct access should free capacity; that is, the amount of capacity that can be used for encoding and maintaining new information should be increased after a retro-cue. We have tested this assumption with a task requiring participants to encode two 
sequential presented memory sets (Souza, Rerko, \& Oberauer). In some trials, a retro-cue was presented in the interval between these sets, pointing to the to-be-probed object from the first arrays. Recognition performance was probed after the second set, separately for each of the two sets. When given a retro-cue that allowed for the removal of irrelevant information from the first set, participants performed better on the recognition task on the second memory set, as is predicted by the removal hypothesis. Providing further evidence for the removal of irrelevant objects from WM, Williams, Hong, Kang, Carlisle, and Woodman (2013) showed that when one out of two memory objects was cued to be forgotten, people had virtually no memory of the to-beforgotten object, as well as significantly improved performance on the remaining relevant object.

Even though the mechanisms behind the three accounts outlined here are different, they are not mutually exclusive: The retro-cue benefit could be accomplished by a combination of these mechanisms - for example, by strengthening the cued representation and removing the noncued WM contents. Similar explanations can probably be formulated within other theoretical frameworks for WM. Our results show that the key to a viable explanation is a distinction between the strength or accessibility of representations in WM-which is modulated by the retro-cue - and the allocation of attention.

Author note This research was supported by a grant from the Swiss National Science Foundation to K.O. (Project No. 100014_126766/1). We thank Iliana Karipidis and Mirko Thalmann for collecting the data.

\section{References}

Astle, D. E., Summerfield, J., Griffin, I., \& Nobre, A. C. (2012). Orienting attention to locations in mental representations. Attention, Perception, \& Psychophysics, 74, 146-162. doi:10.3758/s13414011-0218-3

Awh, E., Vogel, E. K., \& Oh, S.-H. (2006). Interactions between attention and working memory. Neuroscience, 139, 201-208. doi:10.1016/j. neuroscience.2005.08.023

Brainard, D. H. (1997). The Psychophysics Toolbox. Spatial Vision, 10, 433-436. doi:10.1163/156856897X00357

Cowan, N. (2005). Working memory capacity. New York: Psychology Press.

Cowan, N., Saults, J. S., Elliott, E. M., \& Moreno, M. V. (2002). Deconfounding serial recall. Journal of Memory and Language, 46, 153-177.

Downing, P. E., \& Dodds, C. M. (2004). Competition in visual working memory for control of search. Visual Cognition, 11, 689-703.

Farrell, S., \& Lewandowsky, S. (2002). An endogenous distributed model of ordering in serial recall. Psychonomic Bulletin \& Review, 9, 5979. doi:10.3758/BF03196257

Farrell, S., \& Lewandowsky, S. (2012). Response suppression contributes to recency in serial recall. Memory \& Cognition, 40, 1070-1080. doi: $10.3758 / \mathrm{s} 13421-012-0212-6$

Garavan, H. (1998). Serial attention within working memory. Memory \& Cognition, 26, 263-276.
Griffin, I. C., \& Nobre, A. C. (2003). Orienting attention to locations in internal representations. Journal of Cognitive Neuroscience, 15, 1176-1194. doi:10.1162/089892903322598139

Hollingworth, A., \& Maxcey-Richard, A. M. (2013). Selective maintenance in visual working memory does not require sustained visual attention. Journal of Experimental Psychology: Human Perception \& Performance, 39, 1047-1058. doi:10.1037/a0030238

Houtkamp, R., \& Roelfsema, P. R. (2006). The effect of items in working memory on the deployment of attention and the eyes during visual search. Journal of Experimental Psychology: Human Perception and Performance, 32, 423-442. doi:10.1037/0096-1523.32.2.423

Kane, M. J., \& Engle, R. W. (2002). The role of prefrontal cortex in working-memory capacity, executive attention, and general fluid intelligence: An individual-differences perspective. Psychonomic Bulletin \& Review, 9, 637-671. doi:10.3758/BF03196323

Kiyonaga, A., \& Egner, T. (2013). Working memory as internal attention: Toward an integrative account of internal and external selection processes. Psychonomic Bulletin \& Review, 20, 228-242. doi:10. 3758/s13423-012-0359-y

Kuo, B. C., Rao, A., Lepsien, J., \& Nobre, A. C. (2009). Searching for targets within the spatial layout of visual short-term memory. Journal of Neuroscience, 29, 8032-8038. doi:10.1523/ JNEUROSCI.0952-09.2009

Kuo, B. C., Stokes, M., \& Nobre, A. C. (2012). Attention modulates maintenance of representations in visual short-term memory. Journal of Cognitive Neuroscience, 24, 51-61. doi:10.1162/jocn a_ 00087

Landman, R., Spekreijse, H., \& Lamme, A. F. (2003). Large capacity storage of integrated objects before change blindness. Vision Research, 43, 149-164. doi:10.1016/S0042-6989(02)00402-9

LaRocque, J. J., Lewis-Peacock, J. A., Drysdale, A. T., Oberauer, K., \& Postle, B. R. (2013). Decoding attended information in short-term memory: An EEG study. Journal of Cognitive Neuroscience, 25, 127-142. doi:10.1162/jocn_a_00305

Lepsien, J., \& Nobre, A. C. (2007). Attentional modulation of object representations in working memory. Cerebral Cortex, 17, 2072 2083. doi:10.1093/cercor/bhl116

Lewis-Peacock, J. A., Drysdale, A. T., Oberauer, K., \& Postle, B. R. (2012). Neural evidence for a distinction between short-term memory and the focus of attention. Journal of Cognitive Neuroscience, 24, 61-79. doi:10.1162/jocn_a_00140

Loaiza, V. M., \& McCabe, D. P. (2012). Temporal-contextual processing in working memory: Evidence from delayed cued recall and delayed free recall tests. Memory \& Cognition, 40, 191-203. doi:10.3758/ s13421-011-0148-2

Makovski, T., \& Jiang, Y. V. (2007). Distributing versus focusing attention in visual short term memory. Psychonomic Bulletin \& Review, 14, 1072-1078.

Makovski, T., \& Jiang, Y. V. (2008). Proactive interference from items previously stored in visual working memory. Memory \& Cognition, 36, 43-52. doi:10.3758/MC.36.1.43

Makovski, T., Sussman, R., \& Jiang, Y. V. (2008). Orienting attention in visual working memory reduces interference from memory probes. Journal of Experimental Psychology: Learning, Memory, and Cognition, 34, 369-380. doi:10.1037/0278-7393.34.2.369

Matsukura, M., \& Hollingworth, A. (2011). Does visual short-term memory have a high-capacity stage? Psychonomic Bulletin \& Review, 18, 1098-1104. doi:10.3758/s13423-011-0153-2

Matsukura, M., Luck, S. J., \& Vecera, S. P. (2007). Attention effects during visual short-term memory maintenance: Protection or prioritization? Perception \& Psychophysics, 69, 1422-1434. doi:10. 3758/BF03192957

Maxcey-Richard, A. M., \& Hollingworth, A. (2013). The strategic retention of task-relevant objects in visual working memory. Journal of Experimental Psychology: Learning, Memory, and Cognition, 39, 760-772. doi:10.1037/a0029496 
McElree, B. (2006). Accessing recent events. In B. H. Ross (Ed.), The psychology of learning and motivation (Vol. 46, pp. 155-200). San Diego: Academic Press. doi:10.1016/S0079-7421(06)46005-9

Naghavi, H. R., \& Nyberg, L. (2005). Common fronto-parietal activity in attention, memory, and consciousness: Shared demands on integration? Consciousness and Cognition, 14, 390-425.

Nobre, A. C., Coull, J. T., Maquet, P., Frith, C. D., Vandenberghe, R., \& Mesulam, M. M. (2004). Orienting attention to locations in perceptual versus mental representations. Journal of Cognitive Neuroscience, 16, 363-373. doi:10.1162/089892904322926700

Nobre, A. C., Griffin, I. C., \& Rao, A. (2008). Spatial attention can bias search in visual short-term memory. Frontiers in Human Neuroscience, 1(4), 1-9. doi:10.3389/neuro.09.004.2007

Oberauer, K. (2002). Access to information in working memory: Exploring the focus of attention. Journal of Experimental Psychology: Learning, Memory, and Cognition, 28, 411-421. doi: 10.1037/0278-7393.28.3.411

Oberauer, K. (2003). Selective attention to elements in working memory. Experimental Psychology, 50, 257-269.

Oberauer, K. (2005). Control of the contents of working memory-A comparison of two paradigms and two age groups. Journal of Experimental Psychology: Learning, Memory, and Cognition, 31, 714-728. doi:10.1037/0278-7393.31.4.714

Oberauer, K. (2009). Design for a working memory. In B. H. Ross (Ed.), The psychology of learning and motivation: Advances in research and theory (Vol. 51, pp. 45-100). San Diego: Elsevier Academic Press. doi:10.1016/S0079-7421(09)51002-X

Oberauer, K., \& Hein, L. (2012). Attention to information in working memory. Current Directions in Psychological Science, 21, 164-169. doi: $10.1177 / 0963721412444727$

Oberauer, K., Souza, A. S., Druey, M. D., \& Gade, M. (2013). Analogous mechanisms of selection and updating in declarative and procedural working memory: Experiments and a computational model. Cognitive Psychology, 66, 157-211. doi:10.1016/j.cogpsych.2012.11.001

Olivers, C. N. L. (2008). Interactions between visual working memory and visual attention. Frontiers in Bioscience, 13, 1182-1191.

Olivers, C. N. L., Meijer, F., \& Theeuwes, J. (2006). Feature-based memory-driven attentional capture: Visual working memory content affects visual attention. Journal of Experimental Psychology: Human Perception and Performance, 32, 1243-1265. doi:10. 1037/0096-1523.32.5.1243

Olivers, C. N. L., Peters, J., Houtkamp, R., \& Roelfsema, P. R. (2011). Different states in visual working memory: When it guides attention and when it does not. Trends in Cognitive Sciences, 15, 327-334. doi:10.1016/j.tics.2011.05.004

Pelli, D. G. (1997). The VideoToolbox software for visual psychophysics: Transforming numbers into movies. Spatial Vision, 10, 437-442. doi:10.1163/156856897X00366

Pertzov, Y., Bays, P. M., Joseph, S., \& Husain, M. (2013). Rapid forgetting prevented by retrospective attention cues. Journal of Experimental Psychology: Human Perception and Performance, 39, 1224-1231. doi:10.1037/a0030947

Posner, M. I. (1980). Orienting of attention. Quarterly Journal of Experimental Psychology, 32, 3-25. doi:10.1080/ 00335558008248231

Rerko, L., \& Oberauer, K. (2013). Focused, unfocused, and defocused information in working memory. Journal of Experimental Psychology: Learning, Memory, and Cognition, 39, 1075-1096. doi: $10.1037 / \mathrm{a} 0031172$

Schmidt, B. K., Vogel, E. K., Woodman, G. F., \& Luck, S. J. (2002). Voluntary and automatic attentional control of visual working memory. Perception \& Psychophysics, 64, 754-763. doi:10.3758/ BF03194742

Sligte, I. G., Scholte, H. S., \& Lamme, V. A. F. (2008). Are there multiple visual short term memory stores? PLoS ONE, 3, e1699. doi:10.1371/ journal.pone. 0001699

Souza, A. S., Rerko, L., \& Oberauer, K. (2013). Unloading and reloading working memory: Attending to one item frees capacity. Zurich, Switzerland: University of Zurich, Department of Psychology. Unpublished manuscript

Vogel, E. K., Woodman, G. F., \& Luck, S. J. (2001). Storage of features, conjunctions, and objects in visual working memory. Journal of Experimental Psychology: Human Perception and Performance, 27, 92-114. doi:10.1037/0096-1523.27.1.92

Wheeler, M. E., \& Treisman, A. M. (2002). Binding in short-term visual memory. Journal of Experimental Psychology: General, 131, 4864. doi:10.1037/0096-3445.131.1.48

Williams, M., Hong, S. W., Kang, M.-S., Carlisle, N. B., \& Woodman, G. F. (2013). The benefit of forgetting. Psychonomic Bulletin \& Review, 20, 348-355. doi:10.3758/s13423-012-0354-3

Woodman, G. F., \& Vecera, S. P. (2011). The cost of accessing an object's feature stored in visual working memory. Visual Cognition, 19, 112. doi: $10.1080 / 13506285.2010 .521140$

Woodman, G. F., Vecera, M., \& Luck, S. J. (2003). Perceptual organization influences visual working memory. Psychonomic Bulletin \& Review, 10, 80-87. doi:10.3758/BF03196470 